\title{
Performance Analysis and Power Allocation for NOMA-assisted Cloud Radio Access Network
}

\author{
Fangcheng $\mathrm{Xu}^{1}$, Xiangbin $\mathrm{Yu}^{1 *}$, Weiye $\mathrm{Xu}^{2}$, and Jiali Cai ${ }^{1}$ \\ ${ }^{1}$ College of Electronic and Information Engineering, Nanjing University of Aeronautics and Astronautics \\ Nanjing, 210016, China \\ [e-mail: xufangcheng6@163.com, yxbxwy@gmail.com,jiali_cai123@163.com] \\ ${ }^{2}$ School of Information and Communication Engineering, Nanjing Institute of Technology \\ Nanjing, 211167, China \\ [e-mail: xuweiye2014@hotmail.com] \\ *Corresponding author: Xiangbin Yu
}

Received October 5, 2020; revised December 6, 2020; revised January 18, 2021; accepted February 13, 2021; published March 31, 2021

\begin{abstract}
With the assistance of non-orthogonal multiple access (NOMA), the spectrum efficiency and the number of users in cloud radio access network (CRAN) can be greatly improved. In this paper, the system performance of NOMA-assisted CRAN is investigated. Specially, the outage probability (OP) and ergodic sum rate (ESR), are derived for performance evaluation of the system, respectively. Based on this, by minimizing the OP of the system, a suboptimal power allocation (PA) scheme with closed-form PA coefficients is proposed. Numerical simulations validate the accuracy of the theoretical results, where the derived OP has more accuracy than the existing one. Moreover, the developed PA scheme has superior performance over the conventional fixed PA scheme but has smaller performance loss than the optimal PA scheme using the exhaustive search method.
\end{abstract}

Keywords: Cloud Radio Access Networks, Ergodic Sum Rate, Non-Orthogonal Multiple Access, Power Allocation, Outage Probability

This work is partially supported by On-Job Training Doctoral Research Fund of Nanjing Institute of Technology (ZKJ201904), Natural Science Foundation of Jiangsu Province in China (BK20181289), National Natural Science Foundation of China (61571225). 


\section{Introduction}

As next-generation mobile communication system, the 5th-Generation (5G) communication not only has super higher spectrum utilization efficiency and energy efficiency, but also can increase the transmission rate and resource utilization up to one or more magnitudes when compared to the present 4 th-Generation $(4 \mathrm{G})$ mobile communication $[1,2]$.

In particular, non-orthogonal multiple access (NOMA) is a potential multiple access technique in the future communication, and it firstly developed by Japan NTT DoCoMo in 2013 [3]. The core idea of NOMA technique can provide services for multiple users simultaneously by multiplexing the power domain on the same orthogonal resource block (such as time domain, frequency domain, etc.). The existing orthogonal multiple access (OMA) schemes may not meet the requirements of massive connectivity in the future communication scenario, while NOMA can further improve the spectrum utilization and service more users simultaneously $[4,5]$.

To deal with the spectrum scarcity and interference, coordinated multipoint (CoMP) is a promising scheme in the future dense cellular networks. In CoMP, the performance of cell-edge users can be improved by the cooperation of nearby base stations (BSs) [6]. Moreover, generalized coordinated multipoint (GCoMP) technology is proposed to further enhance the overall performance of the network via applying the cooperation of BSs to both of cell-edge and cell-center users [7]. The idea of GCoMP is similar with that of cloud radio access network (CRAN), which reduces the access distance and boosts spectral efficiency by decentralizing the remote radio heads (RRHs) [8,9]. However, the increase of the number of RRHs will restrict the system performance since the available resource blocks will be fewer. To overcome the above shortcoming, NOMA-assisted CRAN (CRAN-NOMA) will become one better scheme, and the resultant higher capacity and spectrum efficiency can be obtained.

In the existing work, the performance of the system with the CRAN was analyzed in [10] by considering the random location of base station (BS), and the outage probability (OP) and ergodic sum rate (ESR) were derived for performance analysis, but the system is limited in single user case. For this reason, on the basis of [10], the authors in [11] studied the OP of CRAN-NOMA, where two paired users are served simultaneously. By means of the performance analysis, an approximate $\mathrm{OP}$ is derived, but it is not accurate enough, especially at low SNR, since some terms in the summation are neglected during the mathematical derivation. Furthermore, the above work does not consider the optimization of power allocation (PA) so that the system performance is not well improved. In fact, the system model in [11] belongs to a special case of GCoMP-enabled NOMA networks in [7]. In [7], the performance of GCoMP-enabled NOMA networks was analyzed and the PA was optimized. However, [7] don't consider the randomness of the distance. Moreover, the PA in [7] requests the instantaneous channel state information (CSI).

Motivated by the above reason, we will study the performance analysis and optimization for CRAN-NOMA system model from the perspective of statistical CSI. Similar to [11], for simplicity, we only focus on the case of one NOMA cluster consisting of two users, while the interferences from other NOMA clusters are not considered. The more generalized performance analysis and optimization for the case of multiple NOMA clusters in CRAN-NOMA networks are the issues worth of research, which will be studied in our future work. Our main contributions are summarized as follows: 
1) By the theoretical analysis, approximate expressions with closed-form for the OPs of a two-user CRAN-NOMA system are derived. Moreover, our simulation demenstrates the derived OP expressions have more accurate than [11].

2) The ESR of the system is also analyzed, then corresponding approximate closed-form expression is derived for evaluation, which is not studied in [11]. It is shown that this theoretical ESR can agree with the simulation result.

3) With the obtained OP, a suboptimal PA solution with the closed-form expression is proposed by minimizing the OP. The propsed PA solution can attain similar performance as the optimal PA solution using one-dimensional (1D) exhaustive search method.

The rest of this paper is organized as follows. The system model of CRAN-NOMA is presented in Section 2. In Section 3, the system performance is analyzed, and the OP and ESR are derived, respectively. In Section 4, suboptimal PA scheme is developed. In Section 5 , the simulation results are provided for evaluation, and conclusions are summarized in Section 6.

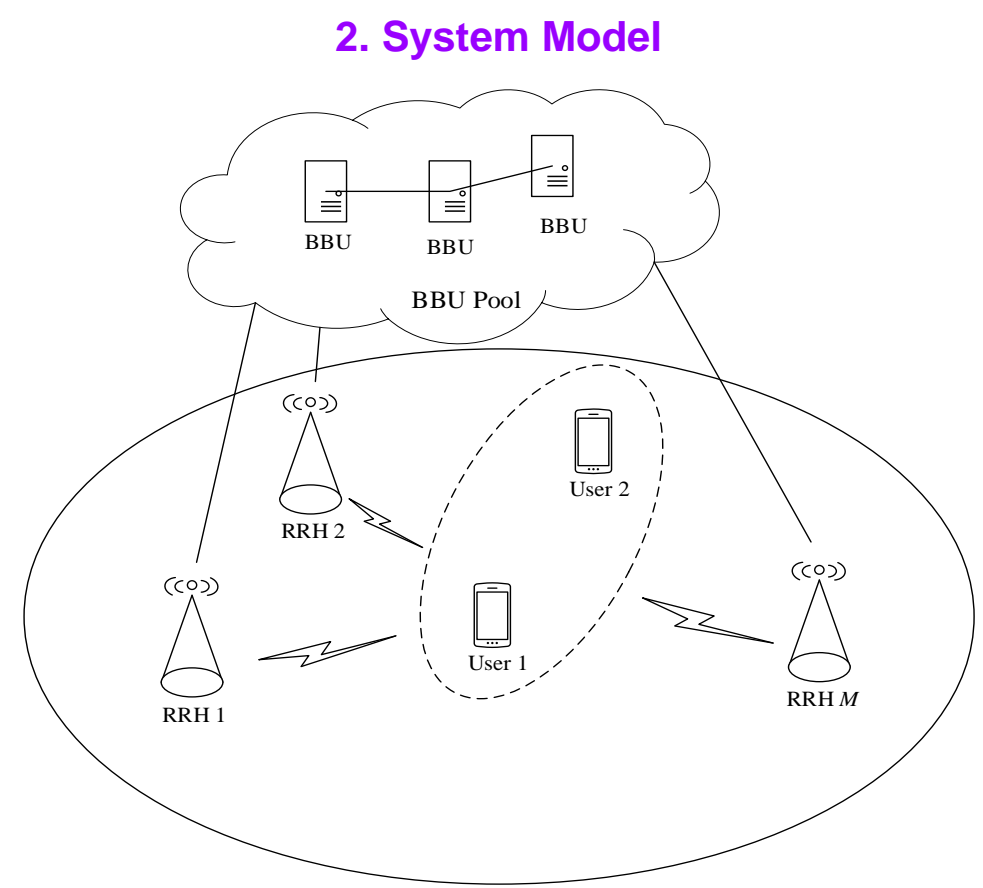

Fig. 1. Downlink CRAN-NOMA system model

Consider a downlink CRAN-NOMA system shown in Fig. 1, where $M$ single-antenna RRHs are distributed in a disk with radius $D$ randomly to serve a NOMA group consisting of two single-antenna users simultaneously, in which user- 1 is located in the center of the disk, and user-2 has the distance of $L(0<L<D)$ to the user-1. For the above CRAN-NOMA system, the channel between the $j$-th RRH $(j=1, \ldots, M)$ and user- $i(i=1,2)$ can be modeled as $[10,11]$

$$
h_{j i}=\frac{g_{j i}}{\sqrt{1+d_{j i}^{\alpha}}},
$$


where $d_{j i}$ represents the distance between the $j$-th RRH and user- $i, \alpha$ is the path-loss exponent, and $g_{j i}$ denotes the corresponding small-scale Rayleigh fading coefficient, i.e., $g_{j i} \sim C N(0,1)$. We denote the channel gain between user- $i$ and the $j$-th RRH as $X_{j i}=\left|h_{j i}\right|^{2}$. Let $S_{M_{i}}=\sum_{j=1}^{M} X_{j i}$ for $i=1,2$, then we have:

$$
S_{M_{i}}=\sum_{j=1}^{M}\left|h_{j i}\right|^{2}=\sum_{j=1}^{M} \frac{\left|g_{j i}\right|^{2}}{1+d_{j i}^{\alpha}},
$$

For downlink, we may assume that each RRH has the same transmission power of $P$, and the $a_{i}(i=1,2)$ stands for the PA coefficient of user- $i$. Besides, $d_{j 1}>d_{j 2}$ may hold for the most of RRHs. This is because user-1 is located at the center of the disk with randomly distributed RRHs [11]. Based on this, and in terms of the principle of NOMA scheme [5], the PA coefficients should satisfy that $a_{1}<a_{2}, a_{1}+a_{2}=1$. Then, the received signal of user- $i$ is given as

$$
y_{i}=\sum_{j=1}^{M} h_{j i}\left(\sqrt{a_{1} P} x_{1}+\sqrt{a_{2} P} x_{2}\right)+n_{i}, i=1,2
$$

where $x_{i}$ represents the message of user- $i$, and $n_{i} \sim C N\left(0, \sigma^{2}\right)$. So, the signal to noise ratio (SNR) is $\rho=P / \sigma^{2}$.

By employing the successive interference cancellation technique, user-1 will firstly detect $x_{2}$ and remove it from the un-decoded signal, then decodes $x_{1}$ without interference of $x_{2}$. Hence, for user- 1 to detect $x_{2}$, the signal to interference plus noise ratio (SINR) is

$$
\gamma_{2 \rightarrow 1}=\frac{a_{2} \rho S_{M_{1}}}{a_{1} \rho S_{M_{1}}+1}
$$

while for user-1 to decode $x_{1}$, the SINR is

$$
\gamma_{1}=a_{1} \rho S_{M_{1}}
$$

Besides, user- 2 decodes its own message $x_{2}$ directly by treating $x_{1}$ as interference. Thus, user-2's SINR is given as

$$
\gamma_{2}=\frac{a_{2} \rho S_{M_{2}}}{a_{1} \rho S_{M_{2}}+1}
$$


According to the results above, the available rates of two users are given by

$$
\left\{\begin{array}{l}
R_{1}=\log _{2}\left(1+\gamma_{1}\right)=\log _{2}\left(1+a_{1} \rho S_{M_{1}}\right), \\
R_{2}=\log _{2}\left(1+\gamma_{2}\right)=\log _{2}\left(1+\frac{a_{2} \rho S_{M_{2}}}{a_{1} \rho S_{M_{2}}+1}\right) .
\end{array}\right.
$$

\section{Performance Analysis of CRAN-NOMA}

In this section, the system performance of CRAN-NOMA system over composite Rayleigh fading channel in terms of the OP and ESR will be analyzed and the corresponding theoretical results will be derived.

\subsection{Outage Probability}

The OP is one of the key indictors to judge outage performance of wireless communication systems over fading channel [10-13], but the related research on CRAN-NOMA is relatively less, and the existing work is not accurate enough. For this reason, we first present the outage performance analysis of CRAN-NOMA over Rayleigh fading channel, and derive the associated OP expressions for assessing the performance.

Let $R_{i}^{o}$ be the target rate for user- $i(i=1,2)$, then user-2's OP is given by

$$
\mathrm{P}_{\mathrm{out}}^{2}=\operatorname{Pr}\left(\gamma_{2}<\delta_{2}\right)=\operatorname{Pr}\left(S_{M_{2}}<\tau\right)
$$

where $\delta_{i}=2^{R_{i}^{o}}-1$ for $i=1,2, \tau=\delta_{2} /\left[\left(a_{2}-a_{1} \delta_{2}\right) \rho\right]$, and the condition $\delta_{2}<a_{2} / a_{1}$ is assumed to guarantee that $\tau>0$ [11]. Besides, user-1's OP is given by [11, 14]:

$$
\mathrm{P}_{\text {out }}^{1}=\operatorname{Pr}\left(\gamma_{1}<\delta_{1}, \gamma_{2 \rightarrow 1}<\delta_{2}\right)=\operatorname{Pr}\left(S_{M_{1}}<\xi\right),
$$

where $\xi=\max (\tau, \beta), \beta=\delta_{1} /\left(a_{1} \rho\right)$. In Appendix, we give the detailed derivation on (9).

By means of the Gauss-Chebyshev integration, an approximate expression for the cumulative density function (CDF) of $S_{M_{1}}$ in $(2)$, denoted by $\hat{F}_{S_{M_{1}}}^{(1)}(w)$, is given as $[10,11]$

$$
\hat{F}_{S_{M_{1}}}^{(1)}(w)=\sum_{t_{1}+t_{2}+\ldots+t_{n}=M} \frac{M !}{t_{1} ! t_{2} ! \ldots . t_{n} !} \sum_{v=1}^{n} \sum_{k=1}^{t_{v}}\left(1+x_{v 1}^{\alpha}\right)^{-k} \phi_{k 1} \frac{\gamma\left(k,\left(1+x_{v 1}^{\alpha}\right) w\right)}{(k-1) !}
$$

where $x_{v 1}=(D / 2)(1+\cos ((2 v-1) /(2 n) \pi)), n$ is an approximate parameter because of using Gauss-Chebyshev integration, $\gamma(a, b)=\int_{0}^{b} t^{a-1} e^{t} d t$ is the lower incomplete gamma function, and 


$$
\phi_{k 1}=\frac{1}{\left(t_{v}-k\right) !}\left\{\frac{d^{\left(t_{v}-k\right)}}{d s^{\left(t_{v}-k\right)}}\left[\left(1+x_{v 1}^{\alpha}+s\right)^{t_{v}} \prod_{r=1}^{n}\left(\frac{\pi}{n D}\left|\sin \frac{2 r-1}{2 n} \pi\right| \frac{x_{r 1}\left(1+x_{r 1}^{\alpha}\right)}{1+x_{r 1}^{\alpha}+s}\right)^{t_{r}}\right]\right\}_{s=-1-x_{v 1}^{\alpha}}
$$

Hence, substituting (10) into (9), user-1's OP can be approximated as

$$
\mathrm{P}_{\mathrm{out}}^{1} \approx \hat{F}_{S_{M_{1}}}^{(1)}(\xi)=\sum_{t_{1}+t_{2}+\ldots+t_{n}=M} \frac{M !}{t_{1} ! t_{2} ! \ldots t_{n} !} \sum_{v=1}^{n} \sum_{k=1}^{t_{v}}\left(1+x_{v 1}^{\alpha}\right)^{-k} \phi_{k 1} \frac{\gamma\left(k,\left(1+x_{v 1}^{\alpha}\right) \xi\right)}{(k-1) !} .
$$

We notice that an approximate closed-form OP expression of user-2 is provided in [11], but the provided expression is not accurate enough since a number of terms are ignored. Based on this, we will derive another approximate expression, which has more accuracy than the expression in [11].

Following the analysis in $[10,11]$, using the Gauss-Chebyshev integration, an approximate expression $\hat{f}_{X_{j 2}}(z)$ for the probability density function (PDF) of $X_{j 2}=\left|h_{j 2}\right|^{2}$ can be derived as

$$
\begin{aligned}
& \hat{f}_{X_{j 2}}(z)=\frac{(D-L) \pi}{n D^{2}} \sum_{v=1}^{n}\left|\sin \left(\frac{2 v-1}{2 n} \pi\right)\right| q\left(x_{v 2}\right)\left(1+x_{v 2}^{\alpha}\right) \\
& +\frac{2 L}{n D^{2}} \sum_{v=1}^{n}\left|\sin \left(\frac{2 v-1}{2 n} \pi\right)\right| p\left(x_{v 3}\right)\left(1+x_{v 3}^{\alpha}\right)
\end{aligned}
$$

where

$$
\left\{\begin{array}{l}
q(x)=x e^{-z\left(1+x^{\alpha}\right)} \\
p(x)=x e^{-z\left(1+x^{\alpha}\right)} \arccos \left(\frac{L^{2}+x^{2}-D^{2}}{2 x L}\right)
\end{array}\right.
$$

and

$$
\left\{\begin{array}{l}
x_{v 2}=\frac{D-L}{2}\left[1+\cos \left(\frac{2 v-1}{2 n} \pi\right)\right] \\
x_{v 3}=L \cos \left(\frac{2 v-1}{2 n} \pi\right)+D
\end{array}\right.
$$

Considering the independent identically distributed (i.i.d.) characterization of $X_{j 2}(j=1, \ldots, M)$, the Laplace transform of $S_{M 2}$ in (2), $L_{S_{M 2}}(s)$ can be approximated as 


$$
\begin{aligned}
& L_{S_{M 2}}(s)=\left[L_{X_{j 2}}(s)\right]^{M} \approx\left[\int_{0}^{\infty} e^{-s z} \hat{f}_{X_{j 2}}(z) d z\right]^{M} \\
& =\sum_{t_{1}+t_{2}+\ldots+t_{n}=M} \frac{M !}{t_{1} ! t_{2} ! \ldots . t_{n} !} \sum_{v=1}^{n} \sum_{k=1}^{t_{v}}\left[\phi_{k 2}\left(1+x_{v 2}^{\alpha}+s\right)^{-k}+\phi_{k 3}\left(1+x_{v 3}^{\alpha}+s\right)^{-k}\right],
\end{aligned}
$$

where

$$
\left\{\begin{array}{l}
\phi_{k 2}=\frac{1}{\left(t_{v}-k\right) !}\left\{\frac{d^{\left(t_{v}-k\right)}}{d s^{\left(t_{v}-k\right)}}\left[\left(1+x_{v 2}^{\alpha}+s\right)^{t_{v}} \times Q_{r}\right]\right\}_{s=-1-x_{v 2}^{\alpha}}, \\
\phi_{k 3}=\frac{1}{\left(t_{v}-k\right) !}\left\{\frac{d^{\left(t_{v}-k\right)}}{d s^{\left(t_{v}-k\right)}}\left[\left(1+x_{v 3}^{\alpha}+s\right)^{t_{v}} \times Q_{r}\right]\right\}_{s=-1-x_{v 3}^{\alpha}},
\end{array}\right.
$$

and

$$
Q_{r}=\prod_{r=1}^{n}\left[\begin{array}{l}
\frac{(D-L) \pi}{n D^{2}}\left|\sin \frac{2 r-1}{2 n} \pi\right| \frac{x_{r 2}\left(1+x_{r 2}^{\alpha}\right)}{1+s+x_{r 2}^{\alpha}} \\
+\frac{2 L}{n D^{2}}\left|\sin \frac{2 r-1}{2 n} \pi\right| \arccos \left(\frac{-D^{2}+x_{r 3}^{2}+L^{2}}{2 x_{r 3} L}\right) \frac{x_{r 3}\left(1+x_{r 3}^{\alpha}\right)}{1+s+x_{r 3}^{\alpha}}
\end{array}\right]^{t_{v}}
$$

In (17) and (18), the derived expressions of $\phi_{k 2}$ and $\phi_{k 3}$ are accurate. It is noticed that Ref.[11] also gives the corresponding derivations, i.e., $\beta_{k 2}$ and $\beta_{k 3}$ in the equation (10) of [11], which are shown as

$$
\beta_{k 2}=\frac{1}{\left(t_{v}-k\right) !}\left\{\frac{d^{\left(t_{v}-k\right)}}{d s^{\left(t_{v}-k\right)}}\left[\left(1+x_{v 2}^{\alpha}+s\right)^{t_{v}} \times \prod_{r=1}^{n}\left(\left|\sin \frac{2 r-1}{2 n} \pi\right| \frac{x_{r 2}\left(1+x_{r 2}^{\alpha}\right)}{1+s+x_{r 2}^{\alpha}}\right)\right]\right\}_{s=-1-x_{v 2}^{\alpha}}
$$

And

$$
\beta_{k 3}=\frac{1}{\left(t_{v}-k\right) !}\left\{\frac{d^{\left(t_{v}-k\right)}}{d s^{\left(t_{v}-k\right)}}\left[\left(1+x_{v 3}^{\alpha}+s\right)^{t_{v}} \times \prod_{r=1}^{n}\left(\left|\sin \frac{2 r-1}{2 n} \pi\right| \arccos \left(\frac{-D^{2}+x_{r 3}^{2}+L^{2}}{2 x_{r 3} L}\right) \frac{x_{r 3}\left(1+x_{r 3}^{\alpha}\right)}{1+s+x_{r 3}^{\alpha}}\right)\right]\right\}_{s=-1-x_{r 3}^{\alpha}} .
$$

So, when compared to (17) and (18), they are not accurate enough because some terms of $Q_{r}$ in (18) are neglected, which result in the inaccuracy of OP in the equation (10) of [11].

By applying the inverse Laplace transform of $L_{S_{M 2}}(s)$, an approximate expression $\hat{f}_{S_{M_{2}}}(z)$ for the PDF of $S_{M 2}$ in (2) is obtained by 


$$
\hat{f}_{S_{M 2}}(z) \approx \sum_{t_{1}+t_{2}+\ldots+t_{n}=M} \frac{M !}{t_{1} ! t_{2} ! \ldots . t_{n} !} \sum_{v=1}^{n} \sum_{k=1}^{t_{v}}\left[\frac{\phi_{k 2}}{(k-1) !} z^{k-1} e^{-\left(1+x_{v 2}^{\alpha}\right) z}+\frac{\phi_{k 3}}{(k-1) !} z^{k-1} e^{-\left(1+x_{v 3}^{\alpha}\right) z}\right]
$$

Thus, with (21), an approximate expression $\hat{F}_{S_{M_{2}}}^{(1)}(z)$ for the CDF of $S_{M 2}$ is given by

$$
\hat{F}_{S_{M_{2}}}^{(1)}(z) \approx \sum_{t_{1}+t_{2}+\ldots+t_{n}=M} \frac{M !}{t_{1} ! t_{2} ! \ldots t_{n} !} \sum_{v=1}^{n} \sum_{k=1}^{t_{v}}\left[\phi_{k 2} \frac{\gamma\left(k,\left(1+x_{v 2}^{\alpha}\right) w\right)}{\left(1+x_{v 2}^{\alpha}\right)^{k}(k-1) !}+\phi_{k 3} \frac{\gamma\left(k,\left(1+x_{v 3}^{\alpha}\right) w\right)}{\left(1+x_{v 3}^{\alpha}\right)^{k}(k-1) !}\right]
$$

Therefore, substituting (22) into (8) yields

$$
\begin{aligned}
& \mathrm{P}_{\mathrm{out}}^{2} \approx \hat{F}_{S_{M_{2}}}^{(1)}(\tau) \\
& =\sum_{t_{1}+t_{2}+\ldots+t_{n}=M} \frac{M !}{t_{1} ! t_{2} ! \ldots t_{n} !} \sum_{v=1}^{n} \sum_{k=1}^{t_{v}}\left[\phi_{k 2} \frac{\gamma\left(k,\left(1+x_{v 2}^{\alpha}\right) \tau\right)}{\left(1+x_{v 2}^{\alpha}\right)^{k}(k-1) !}+\phi_{k 3} \frac{\gamma\left(k,\left(1+x_{v 3}^{\alpha}\right) \tau\right)}{\left(1+x_{v 3}^{\alpha}\right)^{k}(k-1) !}\right.
\end{aligned}
$$

Equation (23) is an approximate closed-form OP expression of user-2. It is shown that Eq.(23) has more accuracy than the equation (10) in [11]. This is because the latter neglects some terms during the derivations of $\beta_{k 2}$ and $\beta_{k 3}$. Simulation results will testify the validity of the (23).

\subsection{Ergodic Sum Rate}

In this subsection, we will derive the theoretical result of ESR in CRAN-NOMA. Using the series expansion of $\gamma(a, b)$, the following equality holds:

$$
\frac{\gamma\left(k,\left(1+x_{v}^{\alpha}\right) w\right)}{(k-1) !}=1-\sum_{l=0}^{k-1} \frac{\left(1+x_{v}^{\alpha}\right)^{l}}{l !} w^{l} e^{-\left(1+x_{v}^{\alpha}\right) w} .
$$

According to the normalization of CDF, that is, $\lim _{w \rightarrow \infty} \hat{F}_{S_{M_{i}}}^{(i)}(w) \approx 1(i=1,2)$, then we have:

$$
\sum_{t_{1}+t_{2}+\ldots+t_{n}=M} \frac{M !}{t_{1} ! t_{2} ! \ldots t_{n} !} \sum_{v=1}^{n} \sum_{k=1}^{t_{v}}\left(1+x_{v 1}^{\alpha}\right)^{-k} \phi_{k 1} \approx 1
$$

and

$$
\sum_{t_{1}+t_{2}+\ldots+t_{n}=M} \frac{M !}{t_{1} ! t_{2} ! \ldots . t t_{n} !} \sum_{v=1}^{n} \sum_{k=1}^{t_{v}}\left[\left(1+x_{v 2}^{\alpha}\right)^{-k} \phi_{k 2}+\left(1+x_{v 3}^{\alpha}\right)^{-k} \phi_{k 3}\right] \approx 1 .
$$

Substituting (24) and (25) into (10) gives another different approximate expression 
$\hat{F}_{S_{M_{1}}}^{(2)}(w)$ for the CDF of $S_{M_{1}}$ in $(2)$ :

$$
\hat{F}_{S_{M_{1}}}^{(2)}(w)=1-\sum_{t_{1}+t_{2}+\ldots+t_{n}=M} \frac{M !}{t_{1} ! t_{2} ! \ldots t_{n} !} \sum_{v=1}^{n} \sum_{k=1}^{t_{v}}\left(1+x_{v 1}^{\alpha}\right)^{-k} \phi_{k 1} \sum_{l=0}^{k-1} \frac{\left(1+x_{v 1}^{\alpha}\right)^{-l}}{l !} w^{l} e^{-\left(1+x_{v 1}^{\alpha}\right) w} .
$$

Similarly, substituting (24) and (26) into (22), another different approximate expression $\hat{F}_{S_{M_{2}}}^{(2)}(w)$ for the CDF of $S_{M_{2}}$ in (2) can be further expressed as

$$
\begin{aligned}
\hat{F}_{S_{M_{2}}}^{(2)}(w)= & 1-\sum_{t_{1}+t_{2}+\ldots+t_{n}=M} \frac{M !}{t_{1} ! t_{2} ! \ldots t_{n} !} \sum_{v=1}^{n} \sum_{k=1}^{t_{v}}\left(1+x_{v 2}^{\alpha}\right)^{-k} \phi_{k 2} \sum_{l=0}^{k-1} \frac{\left(1+x_{v 2}^{\alpha}\right)^{-l}}{l !} w^{l} e^{-\left(1+x_{v 2}^{\alpha}\right) w} \\
& -\sum_{t_{1}+t_{2}+\ldots+t_{n}=M} \frac{M !}{t_{1} ! t_{2} ! \ldots t_{n} !} \sum_{v=1}^{n} \sum_{k=1}^{t_{v}}\left(1+x_{v 3}^{\alpha}\right)^{-k} \phi_{k 3} \sum_{l=0}^{k-1} \frac{\left(1+x_{v 3}^{\alpha}\right)^{-l}}{l !} w^{l} e^{-\left(1+x_{v 3}^{\alpha}\right) w} .
\end{aligned}
$$

With (27) and (7), using the method of integration by parts and the equation EH II 137(3) in [15], we can derive user-1's ESR as

$$
\begin{aligned}
R_{\text {ave }}^{1}=E\left[R_{1}\right] \approx & \frac{a_{1} \rho}{\ln 2} \sum_{t_{1}+t_{2}+\ldots+t_{n}=M} \frac{M !}{t_{1} ! t_{2} ! \ldots t_{n} !} \sum_{v=1}^{n} \sum_{k=1}^{t_{v}}\left(1+x_{v 1}^{\alpha}\right)^{-k} \phi_{k 1} \\
& \times \sum_{l=0}^{k-1}\left(1+x_{v 1}^{\alpha}\right)^{l}\left(a_{1} \rho\right)^{-l-1} \exp \left(\frac{1+x_{v 1}^{\alpha}}{a_{1} \rho}\right) \Gamma\left(-l, \frac{1+x_{v 1}^{\alpha}}{a_{1} \rho}\right),
\end{aligned}
$$

where $E[\cdot]$ denotes the expectation, and $\Gamma(a, b)=\int_{b}^{\infty} t^{a-1} e^{-t} d t$ denotes the upper incomplete gamma function. Similarly, with (28) and (7), by means of the method of integration by parts and the equation EH II 137(3) in [15], we can calculate the ergodic rates of user-2 as

$$
\begin{aligned}
R_{a v e}^{2} & =E\left[R_{2}\right] \approx \frac{a_{2} \rho}{\ln 2} \sum_{t_{1}+t_{2}+\ldots+t_{n}=M} \frac{M !}{t_{1} ! t_{2} ! \ldots t_{n} !} \sum_{v=1}^{n} \sum_{k=1}^{t_{v}}\left(1+x_{v 2}^{\alpha}\right)^{-k} \phi_{k 2} \sum_{l=0}^{k-1} \frac{\left(1+x_{v 2}^{\alpha}\right)^{l}}{a_{2}} \rho^{-l-1} \\
& \times\left[\exp \left(\frac{1+x_{v 2}^{\alpha}}{\rho}\right) \Gamma\left(-l, \frac{1+x_{v 2}^{\alpha}}{\rho}\right)-a_{1}^{-l} \exp \left(\frac{1+x_{v 2}^{\alpha}}{a_{1} \rho}\right) \Gamma\left(-l, \frac{1+x_{v 2}^{\alpha}}{a_{1} \rho}\right)\right] \\
& +\frac{a_{2} \rho}{\ln 2} \sum_{t_{1}+t_{2}+\ldots+t_{n}=M} \frac{M !}{t_{1} ! t_{2} ! \ldots t_{n} !} \sum_{v=1}^{n} \sum_{k=1}^{t_{v}}\left(1+x_{v 3}^{\alpha}\right)^{-k} \phi_{k 3} \sum_{l=0}^{k-1} \frac{\left(1+x_{v 3}^{\alpha}\right)^{l}}{a_{2}} \rho^{-l-1} \\
& \times\left[\exp \left(\frac{1+x_{v 3}^{\alpha}}{\rho}\right) \Gamma\left(-l, \frac{1+x_{v 3}^{\alpha}}{\rho}\right)-a_{1}^{-l} \exp \left(\frac{1+x_{v 3}^{\alpha}}{a_{1} \rho}\right) \Gamma\left(-l, \frac{1+x_{v 3}^{\alpha}}{a_{1} \rho}\right)\right]
\end{aligned}
$$

Therefore, using (29) and (30), the ESR of CRAN-NOMA can be achieved as 


$$
R_{\text {ave }}=R_{\text {ave }}^{1}+R_{\text {ave }}^{2}
$$

Equation (31) is an approximate closed-form ESR expression, and it will have the value close to the corresponding simulation result.

\section{Power Allocation Scheme}

In this section, we develop a suboptimal PA scheme to minimize the OP of the system. Let $J_{1}\left(a_{1}\right)=\mathrm{P}_{\text {out }}^{1}+\mathrm{P}_{\text {out }}^{2}$, and considering that $a_{1}+a_{2}=1$, then the PA problem can be formulated as

$$
\begin{array}{cl}
\min _{a_{1}} & J_{1}\left(a_{1}\right)=\mathrm{P}_{\text {out }}^{1}+\mathrm{P}_{\text {out }}^{2} \\
\text { s.t. } & 0<a_{1} \leq 0.5, \\
& \delta_{2}<\left(1-a_{1}\right) / a_{1}
\end{array}
$$

For the problem (32), we can resort to the one-dimensional (1D) search method to achieve the optimal solution, but the complexity will become much higher since enough small step is needed to find the optimal solution. For this reason, in what follows, we will present a suboptimal PA scheme to solve the problem (32).

From (8) and (9), it is observed that (32) can be divided into the following two sub-problems. The first sub-problem corresponds to the case of $\tau \geq \beta$. For this case, considering that $\tau=\delta_{2} /\left[\left(a_{2}-a_{1} \delta_{2}\right) \rho\right]$ and $\beta=\delta_{1} /\left(a_{1} \rho\right)$, utilizing $a_{1}+a_{2}=1$, we can derive the following inequality as

$$
a_{1} \geq \delta_{1} /\left(\delta_{1}+\delta_{2}+\delta_{1} \delta_{2}\right)
$$

In terms of two constraint conditions of (32), we can obtain:

$$
a_{1} \leq \min \left\{\frac{1}{1+\delta_{2}}, \frac{1}{2}\right\} \text {. }
$$

Hence, for $\tau \geq \beta$, with (33) and (34), the optimization problem (32) is changed to the following sub-problem, i.e.,

$$
\begin{array}{ll}
\min _{a_{1}} & J_{1}\left(a_{1}\right)=F_{S_{M 1}}(\tau)+F_{S_{M 2}}(\tau) \\
\text { s.t. } & \frac{\delta_{1}}{\delta_{1}+\delta_{2}+\delta_{1} \delta_{2}} \leq a_{1} \leq \min \left\{\frac{1}{1+\delta_{2}}, \frac{1}{2}\right\}
\end{array}
$$

where $\tau=\delta_{2} /\left[\left(1-a_{1}-a_{1} \delta_{2}\right) \rho\right] . J_{1}\left(a_{1}\right)$ in (35) is a monotonically increasing function of $a_{1}$ because $\tau, F_{S_{M 1}}(\tau)$ and $F_{S_{M 2}}(\tau)$ are all monotonically increasing functions of $a_{1}$ under this case. Therefore, the optimal solution of (35) is 


$$
a_{1}^{*}=\delta_{1} /\left(\delta_{1}+\delta_{2}+\delta_{1} \delta_{2}\right)
$$

and correspondingly, $a_{2}^{*}=1-a_{1}^{*}$. Besides, if $\delta_{1} /\left(\delta_{1}+\delta_{2}+\delta_{1} \delta_{2}\right)>0.5$, then no PA can be available. So, this case is not considered.

The second sub-problem corresponds to the case of $\tau \leq \beta$. For this case, we can obtain:

$$
a_{1} \leq \delta_{1} /\left(\delta_{1}+\delta_{2}+\delta_{1} \delta_{2}\right)
$$

Considering that $\delta_{1} /\left(\delta_{1}+\delta_{2}+\delta_{1} \delta_{2}\right)<1 /\left(1+\delta_{2}\right)$, the constraint condition of (32) for $\tau \leq \beta$ becomes

$$
0<a_{1} \leq \delta_{1} /\left(\delta_{1}+\delta_{2}+\delta_{1} \delta_{2}\right)
$$

Correspondingly, the problem (32) is changed as

$$
\begin{array}{ll}
\min _{a_{1}} & J_{1}\left(a_{1}\right)=F_{S_{M 1}}(\beta)+F_{S_{M 2}}(\tau) \\
\text { s.t. } & 0<a_{1} \leq \delta_{1} /\left(\delta_{1}+\delta_{2}+\delta_{1} \delta_{2}\right)
\end{array}
$$

However, under this case, it is hard to solve (39) directly since the expressions of $F_{S_{M 1}}(\beta)$ and $F_{S_{M 2}}(\tau)$ are very complicated, which results in the complexity of the objective function $J_{1}\left(a_{1}\right)$. From (12) and (23), we can find that $F_{S_{M 1}}(x)$ and $F_{S_{M 2}}(x)$ are both monotonically increasing function of $x$. So, we have: $F_{S_{M 2}}(\tau) \leq F_{S_{M 2}}(\beta)$.

Let $J_{2}\left(a_{1}\right)=F_{S_{M 1}}(\beta)+F_{S_{M 2}}(\beta)$, then $J_{2}\left(a_{1}\right) \geq J_{1}\left(a_{1}\right)$. Thus, we can optimize the upper bound of $J_{1}\left(a_{1}\right), J_{2}\left(a_{1}\right)$ to obtain suboptimal solution. Correspondingly, the problem (39) is changed as

$$
\begin{array}{cl}
\min _{a_{1}} & J_{2}\left(a_{1}\right)=F_{S_{M 1}}(\beta)+F_{S_{M 2}}(\beta) \\
\text { s.t. } & 0<a_{1} \leq \delta_{1} /\left(\delta_{1}+\delta_{2}+\delta_{1} \delta_{2}\right)
\end{array}
$$

Since $\beta=\delta_{1} /\left(a_{1} \rho\right), \beta$ is a monotonically decreasing function of $a_{1}$, while $F_{S_{M 1}}(\beta)$ and $F_{S_{M 2}}(\beta)$ are both monotonically increasing function of $\beta$. Therefore, to obtain the minimum of $J_{2}\left(a_{1}\right), a_{1}$ must take the maximum value. Namely, the optimal value of $a_{1}$ is $a_{1}^{o}=\delta_{1} /\left(\delta_{1}+\delta_{2}+\delta_{1} \delta_{2}\right)$ for (40). This solution is also a suboptimal one of the problem (39) because it is obtained by optimizing the upper bound of the objective function in (39).

According to the above results, and considering (36), we can get a suboptimal PA solution as follows: 


$$
\left\{\begin{array}{l}
\hat{a}_{1}^{*}=\frac{\delta_{1}}{\delta_{1}+\delta_{2}+\delta_{1} \delta_{2}}, \\
\hat{a}_{2}^{*}=\frac{\delta_{2}+\delta_{1} \delta_{2}}{\delta_{1}+\delta_{2}+\delta_{1} \delta_{2}} .
\end{array}\right.
$$

\section{Simulation Results and Analysis}

In this section, we will use the computer simulation to assess the effectiveness of the derived theoretical results and the suboptimal PA scheme. The theoretical OPs of user- 1 and user- 2 are calculated by (12) and (23), respectively. The theoretical ESR is computed by (31). Based on the works in $[10,11,16]$, we consider the application of dense homogeneous cellular networks in which the small RRHs are deployed with high intensity that may be greater than the intensity of the users. Specially, we consider a disk containing $M$ RRHs and a NOMA cluster with two users, where our theoretical analysis results can be applicable to this simulation environment. Therefore, the default simulation parameters are set as: disk radius $D=10 \mathrm{~m}$, the distance between two user $L=8 \mathrm{~m}$, the value of approximate parameter in theoretical formulae $n=10$. The number of RRHs $M=3,4$, the path loss factor $\alpha=2$, the minimum rate constraints $R_{1}^{o}=0.5 \mathrm{bit} / \mathrm{s} / \mathrm{Hz}, R_{2}^{o}=1 \mathrm{bit} / \mathrm{s} / \mathrm{Hz}$. The power allocation coefficients are computed by (41), i.e., $a_{1}=0.2265, a_{2}=0.7735$. The supposed environment can be regarded as a small part of the ultra-dense network (UDN) [17], in which access points (APs) have a coverage range of about $10 \mathrm{~m}$, and thousands of APs in $1 \mathrm{~km}^{2}$, while cell range is more than $500 \mathrm{~m}$, and generally less than about $3-5 \mathrm{BSs}$ in $1 \mathrm{~km}^{2}$ in the conventional cellular network.

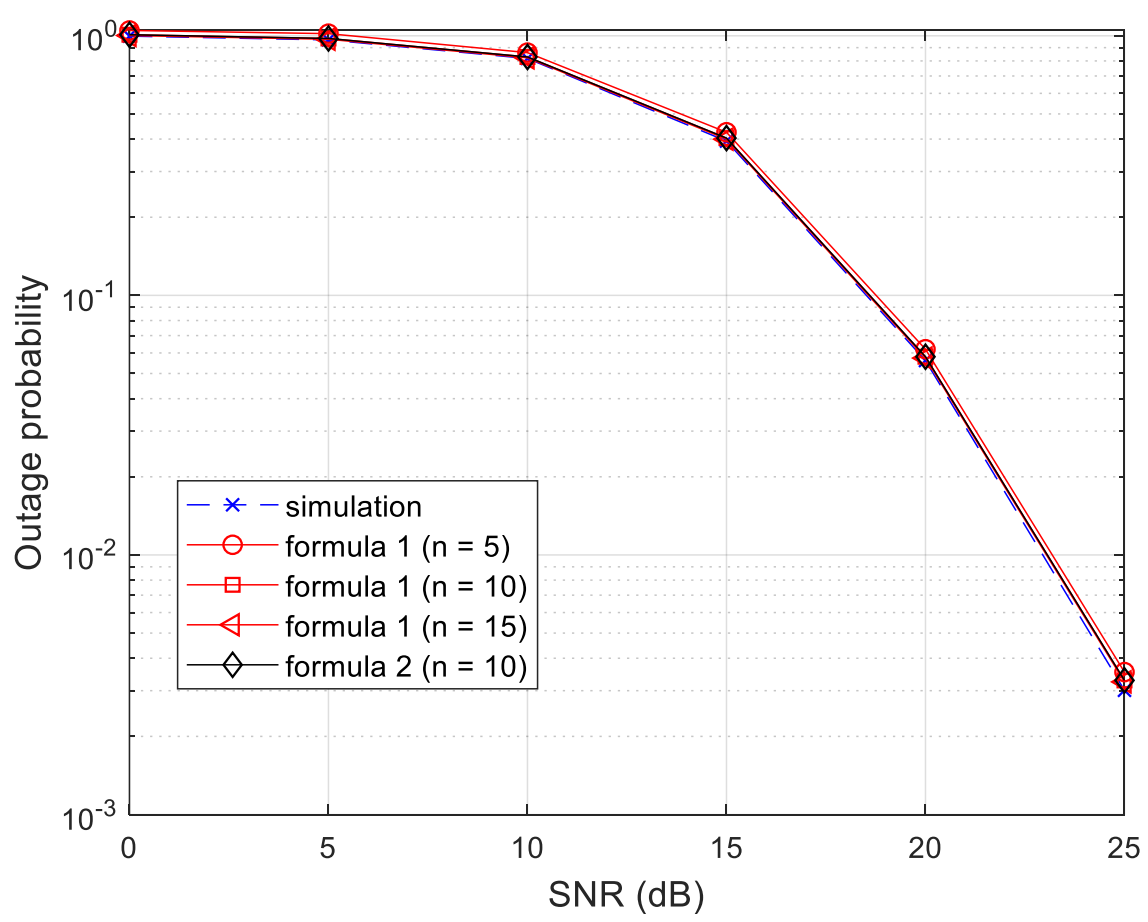

Fig. 2. (a) user-1's OP 
In Fig. 2, we study the outage performance of CRAN-NOMA with different $n$, where the OPs of user-1 and user-2 are assessed respectively in Fig. 2(a) and Fig. 2(b). The theoretical formula 1 is from our paper, i.e., (12) for user-1 and (23) for user-2, whereas the theoretical formula 2 is from [11]. As shown in Fig. 2, as $n$ increases, the accuracy of the theoretical formula 1 is increased as well, and corresponding theoretical value becomes very close to the simulated one, which accords with the characteristic of Gauss-Chebyshev integral method. It can be seen that when $n=10$, the curve of theoretical formula 1 is almost the same as the simulation curve. In fact, when the value of $n$ tends to infinity, the theoretical formula 1 will have the same value as the simulation result. On this account, we can choose $n=10$ for theoretical approximate calculation. Besides, the theoretical formula 2 in [11] for calculating user-1's OP has almost the same value as the simulation result, but the theoretical formula 2 for calculating user-2's OP is different with the corresponding simulation result, especially at low SNR. This is because it neglects a number of terms in the summation during the computation. As a result, the theoretical value is smaller than the actual simulated one. While for our theoretical formula 1, it can compute the OPs of user-1 and user-2 accurately, and agrees well with the corresponding simulation. These results show that our theoretical formulae are valid and can evaluate the system outage performance effectively.

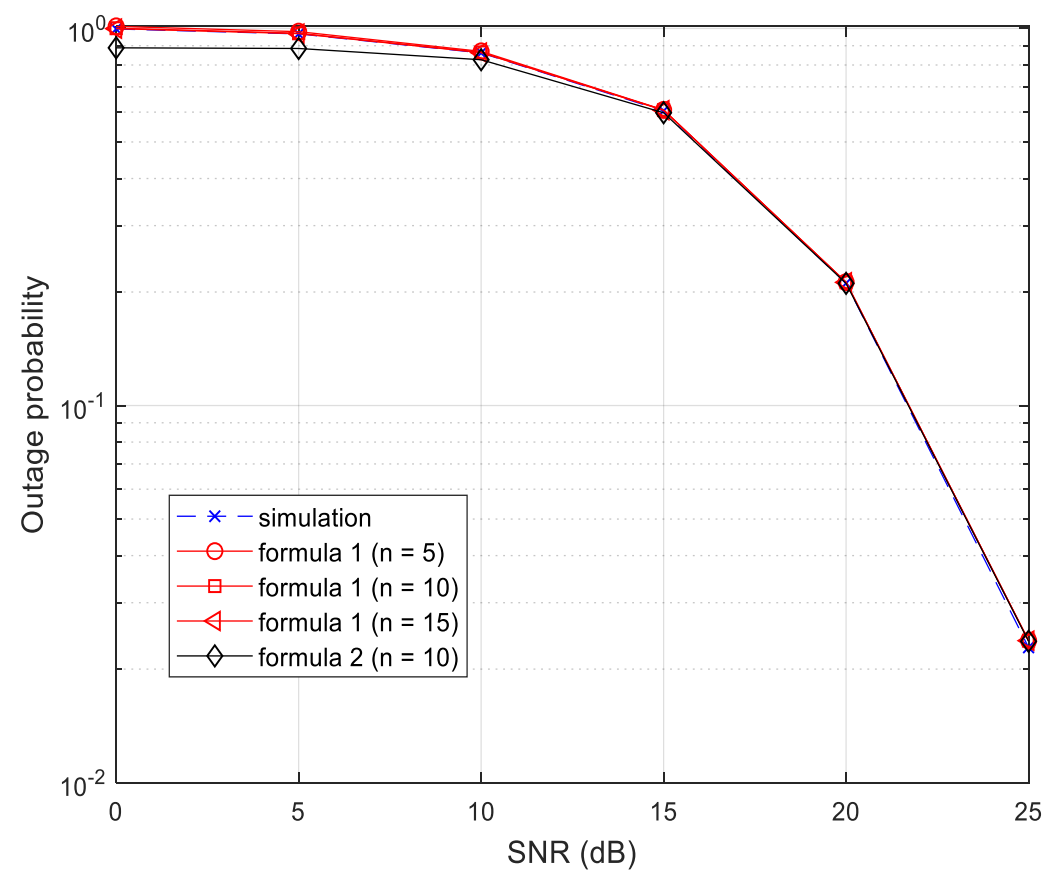

Fig. 2. (b) user-2's OP

Fig. 3 gives the ESR of CRAN-NOMA system, where different values of $M$ and $n$ are considered. From Fig. 3, the ESR increases as $M$ increases. The reason is that when $M$ increases, more RRH can provide users with higher quality of service. Correspondingly, the system OP will be smaller, and resultant ESR will be improved greatly. As expected, the system with $M=5$ has higher ESR than that with $M=3$. Besides, with the increase of the value of $n$, the theoretical ESR becomes more accurate, and the corresponding value is very close to that of the simulation. The above results indicate that our theoretical expression of ESR is also effective. It is worth noting that the Gauss-Chebyshev integration is an 
approximate expression for the original integration confer [18], which means that different approximate expressions for the CDFs of $S_{M_{i}}(i=1,2)$ are used in the derivation of OP and ESR respectively, i.e., (10) and (27) are not equal, (22) and (28) are not equal too. As a result, it may seem that Fig. $\mathbf{2}$ is against Fig. 3, but this phenomenon can be also found in the simulation of [10].

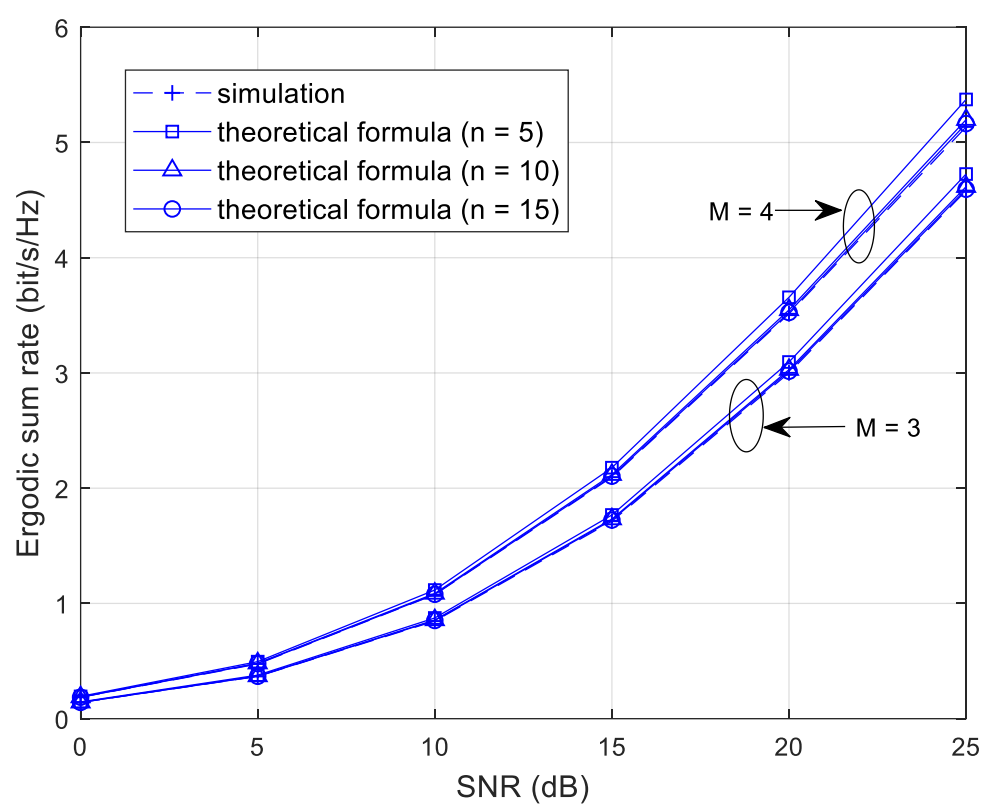

Fig. 3. ESR of CRAN-NOMA system

In Fig. 4, we study the outage performance of CRAN-NOMA with different PA schemes, where the proposed PA scheme, the optimal PA scheme with 1D search method, and two fixed PA schemes are compared. For the first fixed scheme (referred as 'fixed PA 1' in Fig. 4), the PA coefficients are $a_{1}=0.45$ and $a_{2}=0.55$, i.e., the powers of two users are allocated almost the same. For the second fixed scheme (referred as 'fixed PA 2' in Fig. 4), the PA coefficients $a_{1}=0.05$ and $a_{2}=0.95$, i.e., the power allocation has obvious difference, more power is allocated to user-2. It is shown in Fig. 4 that our PA scheme attains similar performance as the optimal one, only with small performance loss at large SNR. Moreover, the optimal PA scheme needs exhaustive search, which will bring about higher complexity. Whereas for our scheme, it has closed-form power allocation, so that its complexity becomes lower. Besides, for low SNR, the four schemes have almost the same performance. The reason is that the noise is the main factor affecting the performance under this case, and resulting OPs of the two users become large. So, the system outage is easy to occur, and the corresponding OP is large. When the SNR is high, the effect of the noise is much less, while the effect of power control becomes much obvious. Thus, the suboptimal scheme and the optimal scheme have better performance than two fixed PA scheme, i.e., they have lower OP than the latter two. This is because the former two can work well with different channel parameters, while for the latter two schemes, the power allocation is fixed, which limits the outage performance. Since the PA coefficients of the fixed PA2 are closer to the suboptimal PA coefficients given by (41) when compared to the PA coefficients of the fixed PA 1 . Hence, the fixed PA 2 has superior performance over the fixed PA 1. The above results demonstrate that our suboptimal PA scheme is reasonable and also efficient. 


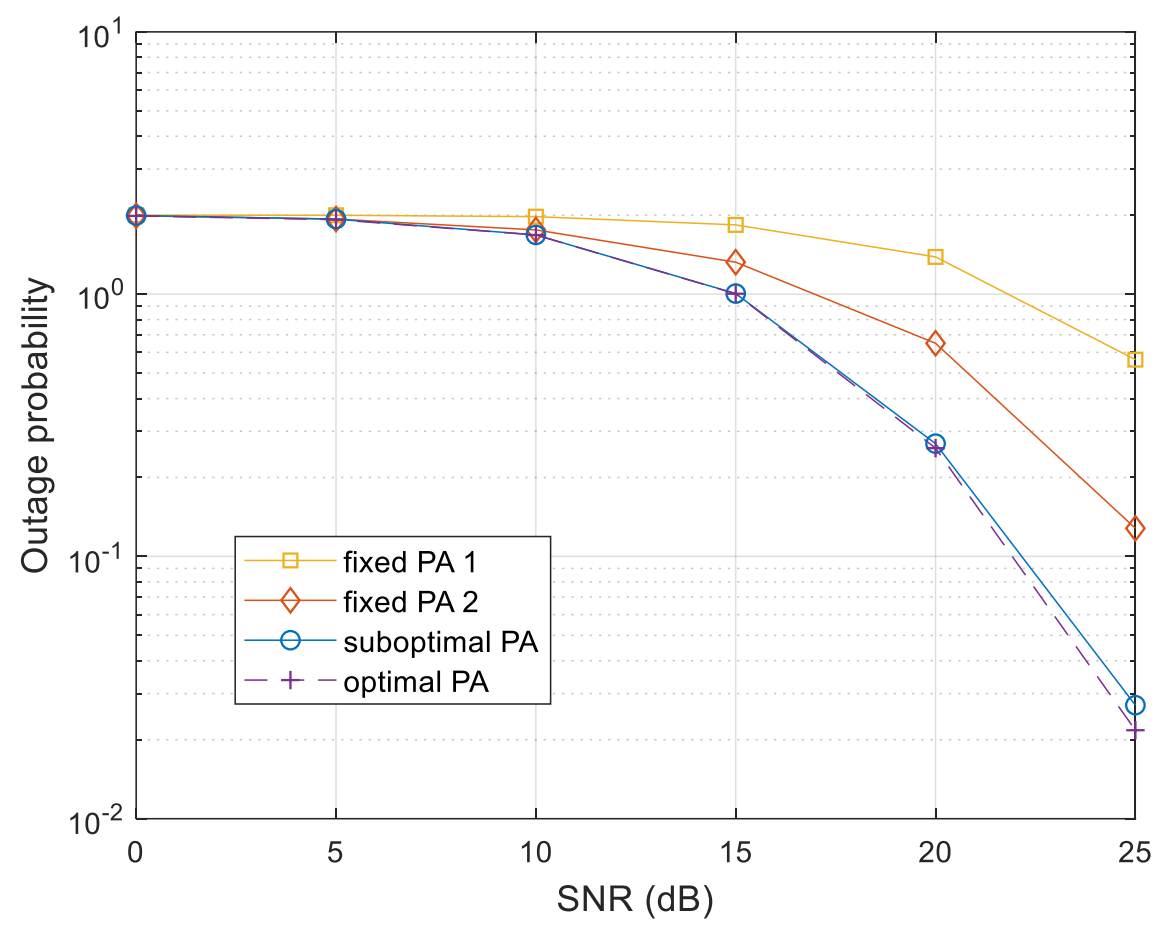

Fig. 4. CRAN-NOMA's OP with different PA schemes

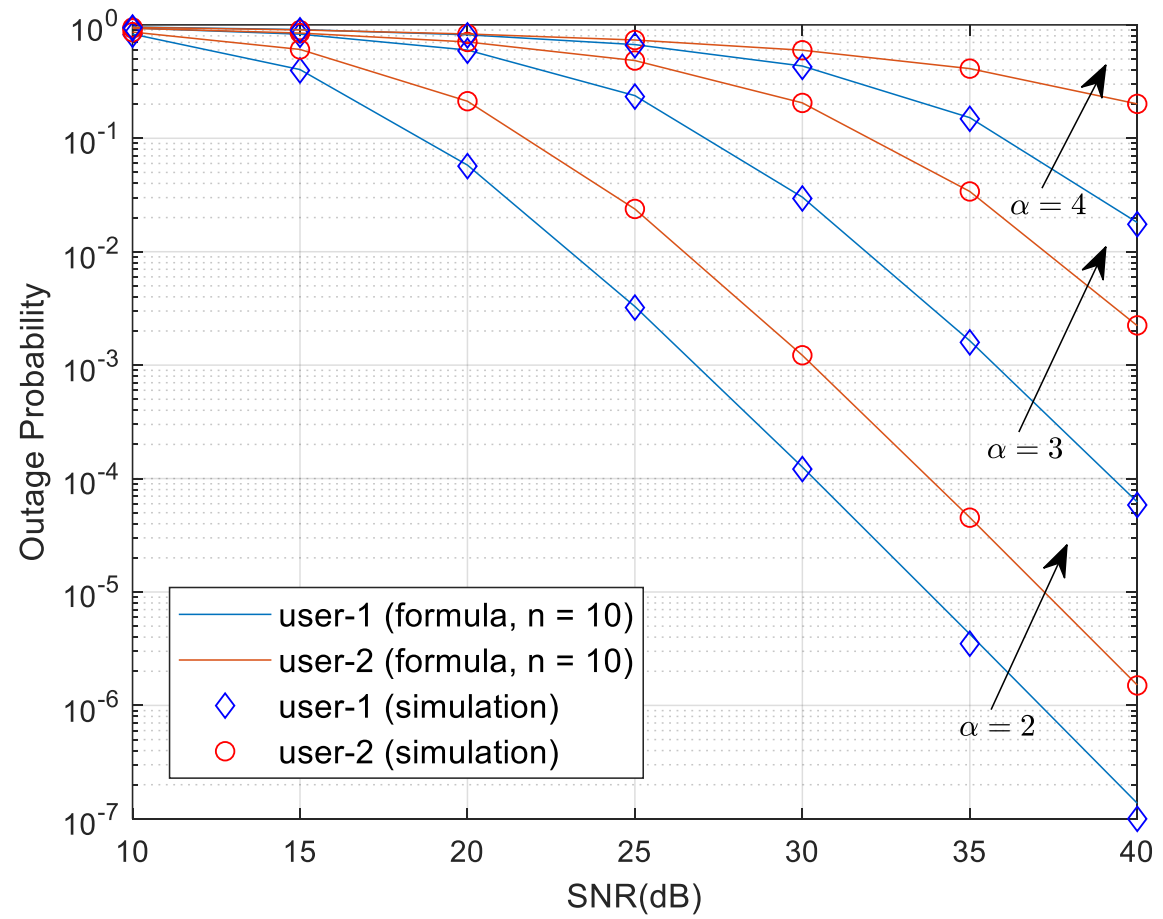

Fig. 5. The OPs of two users with different path loss exponent $\alpha$ 
Fig. 5 plots the OPs of two users in CRAN-NOMA with different path loss exponent $\alpha$, where $\alpha=2,3,4$. Firstly, under different $\alpha$, the theoretical curves are almost coincided with the corresponding simulation curves, thus the accuracy of the proposed approximate theoretical formulas are verified again, and it is shown that the calculation complexity and accuracy can be well balanced for $n=10$. In addition, when $\alpha$ becomes larger, the OPs of two users increases obviously, which indicates that the channel gain greatly affects the achievable rate of users and the corresponding OPs.

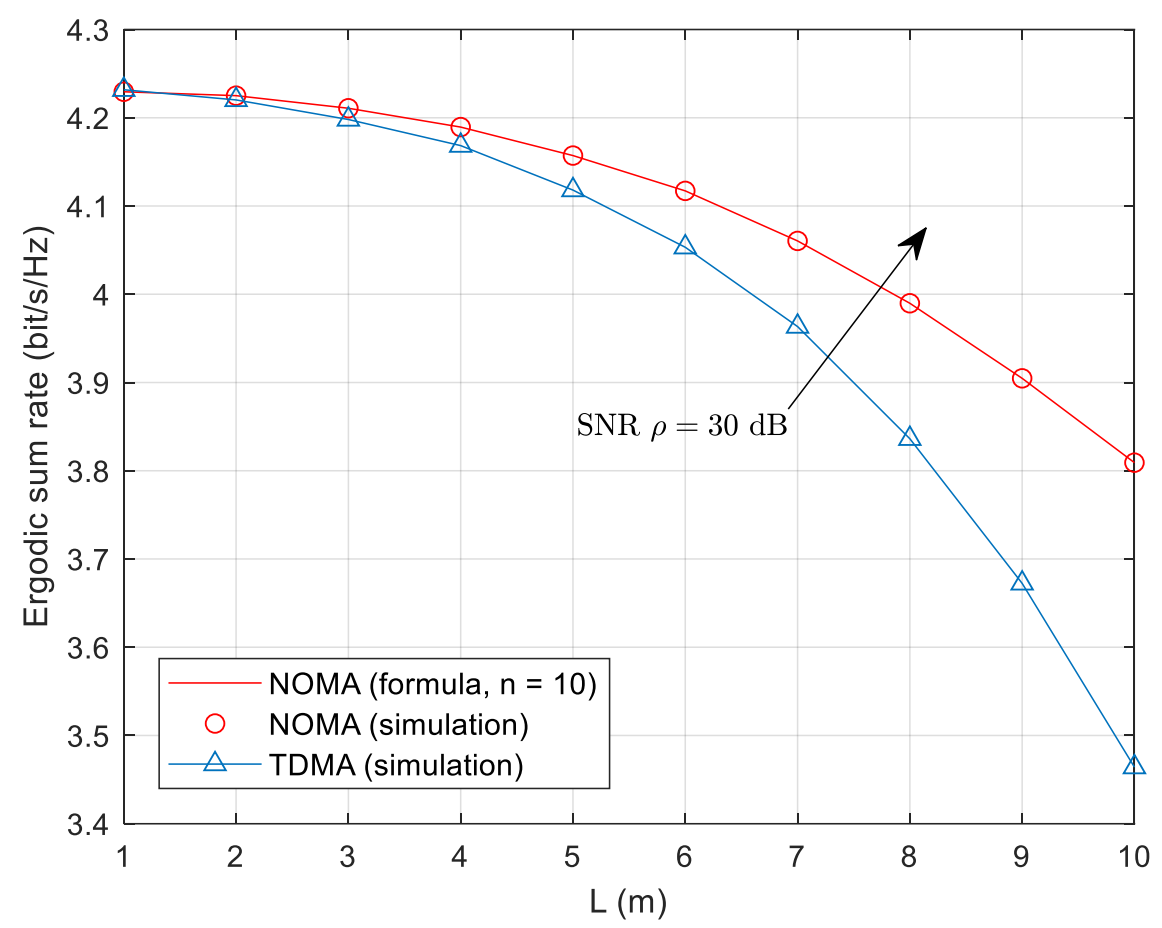

Fig. 6. Comparison of ESR under NOMA and TDMA

Fig. 6 compares the ESR under NOMA and TDMA with varying the distance $L$ between user- 1 and user- 2 , where $a_{1}=0.25, a_{2}=0.75$ in NOMA and two users are allocated with equal time slot in TDMA. It can be verified by Fig. 6 that NOMA can exhibit superior ESR performance than the conventional TDMA due to the power-domain multiplexing gain. Moreover, the performance gap between NOMA and TDMA becomes larger as $L$ increases. This is because the larger $L$ is, the larger the difference of channel gain between two users is, which facilitates the use of NOMA.

\section{Conclusions}

We have studied the performance and power allocation for the CRAN-NOMA system in composite Rayleigh fading channel. With the performance analysis, using the Gauss-Chebyshev integral approximation and the inverse Laplace transform, the OP and ESR of the system are deduced, and the resulting closed-form approximate expressions are attained. The derived OP has more accuracy than the existing one, especially at low SNR. Simulation results verify the correctness of our theoretical OP and ESR. Besides, based on 
the obtained OP, we developed a suboptimal PA scheme with closed-from coefficients to minimize the OP. It is shown that the suboptimal scheme has superior performance over the conventional fixed PA scheme, and can attain similar performance as the optimal 1D search method. Moreover, our PA scheme enjoys lower complexity than the latter since the latter needs larger number of search while our PA scheme has a closed-form expression.

\section{Appendix}

In this appendix, we will present the derivation of (9). With (4) and (5) as well as (7), user-1's OP can be expressed as

$$
\begin{aligned}
\mathrm{P}_{\text {out }}^{1} & =\operatorname{Pr}\left(\left(R_{1}<R_{1}^{o}\right) \cup\left(R_{2 \rightarrow 1}<R_{2}^{o}\right)\right)=1-\operatorname{Pr}\left(\left(R_{1} \geq R_{1}^{o}\right) \cap\left(R_{2 \rightarrow 1} \geq R_{2}^{o}\right)\right), \\
& =1-\operatorname{Pr}\left(\left(\gamma_{1} \geq \delta_{1}\right) \cap\left(\gamma_{2 \rightarrow 1} \geq \delta_{2}\right)\right)
\end{aligned}
$$

where $R_{2 \rightarrow 1}=\log _{2}\left(1+\gamma_{2 \rightarrow 1}\right), \delta_{1}=2^{R_{1}^{o}}-1$, and $\delta_{2}=2^{R_{2}^{o}}-1$.

Let $\tau=\delta_{2} /\left[\left(a_{2}-a_{1} \delta_{2}\right) \rho\right]$ and $\beta=\delta_{1} /\left(a_{1} \rho\right)$, using (4) and (5), then (A1) can be given by

$$
\begin{aligned}
\mathrm{P}_{\text {out }}^{1} & =1-\operatorname{Pr}\left(\left(S_{M_{1}} \geq \delta_{1} /\left(a_{1} \rho\right)\right) \cap\left(S_{M_{1}} \geq \delta_{2} /\left[\left(a_{2}-a_{1} \delta_{2}\right) \rho\right]\right)\right) \\
& =1-\operatorname{Pr}\left(\left(S_{M_{1}} \geq \beta\right) \cap\left(S_{M_{1}} \geq \tau\right)\right)
\end{aligned}
$$

Let $\xi=\max (\tau, \beta)$, then (A2) can be further expressed as

$$
\mathrm{P}_{\text {out }}^{1}=1-\operatorname{Pr}\left(S_{M_{1}} \geq \xi\right)=\operatorname{Pr}\left(S_{M_{1}}<\xi\right)
$$

\section{References}

[1] Q. C. Li, H. Niu, A. T. Papathanassiou, and G. Wu, "5G Network Capacity: Key Elements and Technologies," IEEE Vehicular Technology Magazine, vol. 9, no. 1, pp. 71-78, Mar. 2014. Article (CrossRef Link)

[2] M. Shafi, A. F. Molisch, P. J. Smith, T. Haustein, P. Zhu, P. D. Silva, F. Tufvesson, A. Benjebbour, and G. Wunder, "5G: A Tutorial Overview of Standards, Trials, Challenges, Deployment, and Practice," IEEE Journal on Selected Areas in Communications, vol. 35, no. 6, pp. 1201-1221, June 2017. Article (CrossRef Link)

[3] Y. Saito, Y. Kishiyama, A. Benjebbour, T. Nakamura, A. Li, and K. Higuchi, "Non-Orthogonal Multiple Access (NOMA) for Cellular Future Radio Access," in Proc. of IEEE $77^{\text {th }}$ Vehicular Technology Conference (VTC Spring), pp. 1-5, 2013. Article (CrossRef Link)

[4] L. Dai, B. Wang, Y. Yuan, S. Han, I. Chih-lin, and Z. Wang, "Non-Orthogonal Multiple Access for 5G: Solutions, Challenges, Opportunities, and Future Research Trends," IEEE Communications Magazine, vol. 53, no. 9, pp. 74-81, Sep. 2015. Article (CrossRef Link)

[5] Z. Ding, Z. Yang, P. Fan, and H. V. Poor, "On the Performance of Non-Orthogonal Multiple Access in 5G Systems with Randomly Deployed Users," IEEE Signal Processing Letters, vol. 21, no. 12, pp. 1501-1505, Dec. 2014. Article (CrossRef Link) 
[6] W. Shin, M. Vaezi, B. Lee, D. J. Love, J. Lee, and H. V. Poor, "Non-Orthogonal Multiple Access in Multi-Cell Networks: Theory, Performance, and Practical Challenges," IEEE Communications Magazine, vol. 55, no. 10, pp. 176-183, Oct. 2017. Article (CrossRef Link)

[7] Y. Al-Eryani, E. Hossain, and D. I. Kim, "Generalized Coordinated Multipoint (GCoMP)-Enabled NOMA: Outage, Capacity, and Power Allocation," IEEE Transactions on Communications, vol. 67, no. 11, pp. 7923-7936, Nov. 2019. Article (CrossRef Link)

[8] A. Checko, H. L. Christiansen, Y. Yan, L. Scolari, G. Kardaras, M. S. Berger, and L. Dittmann, "Cloud RAN for Mobile Networks-A Technology Overview," IEEE Communications Surveys \& Tutorials, vol. 17, no. 1, pp. 405-426, 2015. Article (CrossRef Link)

[9] T. X. Tran, A. Hajisami, and D. Pompili, "Cooperative Hierarchical Caching in 5G Cloud Radio Access Networks," IEEE Network, vol. 31, no. 4, pp. 35-41, 2017. Article (CrossRef Link)

[10] Z. Yang, Z. Ding and P. Fan, "Performance Analysis of Cloud Radio Access Networks with Uniformly Distributed Base Stations," IEEE Transactions on Vehicular Technology, vol. 65, no. 1, pp. 472-477, Jan. 2016. Article (CrossRef Link)

[11] X. Gu, X. Ji, Z. Ding, W. Wu, and M. Peng, "Outage Probability Analysis of Non-Orthogonal Multiple Access in Cloud Radio Access Networks," IEEE Communications Letters, vol. 22, no. 1, pp. 149-152, Jan. 2018. Article (CrossRef Link)

[12] W. Xu, Q. Wang, Y. Wang, and B. Wu, "Downlink Performance of Distributed Antenna Systems in MIMO Composite Fading Channel," KSII Transactions on Internet and Information Systems, vol. 8, no. 10, pp. 3342-3360, 2014. Article (CrossRef Link)

[13] L. Lv, J. Chen, Q. Ni, Z. Ding, and H. Jiang, "Cognitive Non-Orthogonal Multiple Access with Cooperative Relaying: A New Wireless Frontier for 5G Spectrum Sharing," IEEE Communications Magazine, vol. 56, no. 4, pp. 188-195, Apr. 2018. Article (CrossRef Link)

[14] J. Men and J. Ge, "Performance Analysis of Non-Orthogonal Multiple Access in Downlink Cooperative Network," IET Communications, vol. 9, no. 18, pp. 2267-2273, 2015. Article (CrossRef Link)

[15] A. Jeffrey and D. Zwillinger, Table of Integrals, Series, Products, $7^{\text {th }}$ Ed, New York, NY, USA: Academic Press, 2007.

[16] Z. Ding and H. V. Poor, "The Use of Spatially Random Base Stations in Cloud Radio Access Networks," IEEE Signal Processing Letters, vol. 20, no. 11, pp. 1138-1141, Nov. 2013. Article (CrossRef Link)

[17] S. Chen, F. Qin, B. Hu, X. Li, and Z. Chen, "User-centric ultra-dense networks for 5G: challenges, methodologies, and directions," IEEE Wireless Communications, vol. 23, no. 2, pp. 78-85, Apr. 2016. Article (CrossRef Link)

[18] F. B. Hildebrand, Introduction to Numerical Analysis, NY, USA: Dover Publications, 1987. 


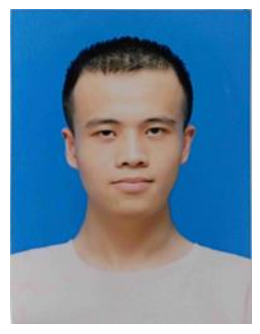

Fangcheng $\mathrm{Xu}$ received his B.S. degree in Information Engineering from Nanjing University of Aeronautics and Astronautics. He is currently working towards the M.Sc. degree at Nanjing University of Aeronautics and Astronautics.

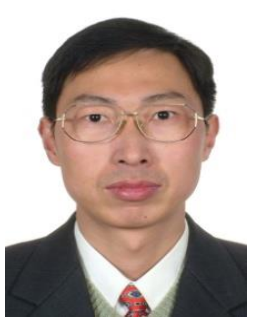

Xiangbin Yu received his Ph.D. in Communication and Information Systems in 2004 from National Mobile Communications Research Laboratory at Southeast University, China. He is currently a Full Professor with the Nanjing University of Aeronautics and Astronautics, China. From 2014 to 2015, he worked as a Visiting Scholar in the Electrical and Computer Engineering, University of Delaware, USA. He has been Senior Member of the Chinese Institute of Electronics since 2012. Dr. Yu served as a technical program committee member of the 2006 and 2017 IEEE Global Telecommunications Conference, and the 2015 and 2018 IEEE International Conference on Communications. He is also a Reviewer for several journals. His research interests include distributed MIMO, mmWave communication, precoding, and green communication.

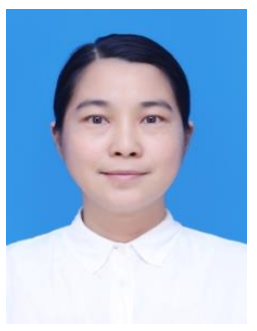

Weiye $\mathrm{Xu}$ received the B.S. degree in Communication Engineering and the M.S. degree in Communication and Information Systems from Hohai University, Nanjing, China, in 2001 and 2007, respectively. She received Ph.D. degree in Information and Communication Engineering from Army Engineering University of PLA, Nanjing, China, in 2019. During 2019-2020, she serves as a Visiting Scholar with the School of Informatics, University of Leicester, U.K. She is currently a associate professor with the School of Information and Communication Engineering, Nanjing Institute of Technology, Nanjing, China. Her research interests emphasize on digtial communication, MIMO technique, distributed antenna systems.

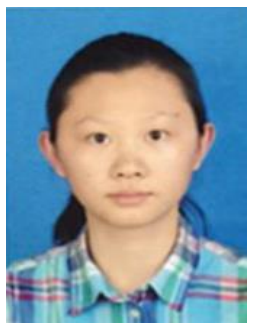

Jiali Cai received her B.S. degree in Communication Engineering from Zhejiang University of Technology. She is currently working towards the M.Sc. degree at Nanjing University of Aeronautics and Astronautics. 